\title{
Fundamentalabbildung und Potentialbestimmung gegebener Riemannscher Flächen.
}

\author{
Von \\ Paul Koebe in Jena.
}

Es sei $F$ ein endlich-vielblättriger, endlich-vielfach zusammenhängender, analytisch begrenzter Bereich mit endlich vielen Windungspunkten im Innern. Während die alten Methoden die Abbildungsaufgabe als Anwendung potentialtheoretischer ${ }^{1}$ ) Entwicklungen behandeln, gestatten die neuen, rein funktionentheoretischen Methoden der konformen Abbildung eine Umkehrung der Reihenfolge der Problemstellungen vorzunehmen ${ }^{1 a}$ ).

\section{Ränderzuordnung.}

Wir können die Fundamentalabbildung der Fläche $F$ rein funktionentheoretisch ausführen, d. i. die eineindeutige konforme Abbildung der zu $F$ gehörenden einfach zusammenhängenden relativ unverzweigten Úberagerungsfläche $F^{(x)}$ auf die Fläche eines $\zeta$-Einheitskreises. Denken wir uns diese Abbildung zunächst nur für die inneren Punkte des Bereichs erklärt, so kann nunmehr durch eine besondere Untersuchung dargetan werden, daß die Abbildungsfunktion $\zeta(z)$ auch längs der Randlinien regulär, in den Eckpunkten zwischen den einzelnen regulär analytischen Begrenzungsteilen jedenfalls stetig ist. Dies ergibt sich unter Einführung einer bemerkenswerten Modifikation gegenüber meinen früheren Entwicklungen zur Frage der Ränderzuordnung ${ }^{9}$ ) bei analytisch oder allgemeiner stetig begrenzten Bereichen folgendermaßen.

1) Riemann-Hilbert: Dirichletsehes Prinzip; Schwarz und C. Neumann: Alternierendes Verfahren; Poincaré: Methode de balayage.

12). Vgl. eine Bemerkung am Schlusse meines Artikels, „Uber eine neue Methode der konformen Abbildung und Uniformieierung", Gött. Nachr. 1912, S. 848.

2) S. meinen Artikel, „Ränderzuordnung bei konformer Abbildung“, Gött. Nachr.

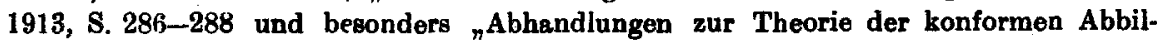
dung. $I^{\mu}$, dritter. Teil, Journ f. Math. 145 (1915), S. 205-219. 
Die Fläche $F$ kann als Teil einer Fläche $F^{\prime}$ aufgefaßt werden, die aus $F$ durch äußere Anfügung zweifach zusammenhängender schmaler Flächenstreifen entsteht. Die Funktion $\zeta^{\prime}(z)$, die die Fundamentalabbildung der Fläche $F^{\prime}$ leistet, liefert gleichzeitig eine völlig reguläre Obertragung der Fläche $F^{(x)}$ auf einen schlichten einfach zusammenhängenden Bereich $\Phi$. Die Untersuchung der Ränderzuordnung für die Fundamentalabbildung der Fläche $F$ ist dadurch auf die Untersuchung der Ränderzuordnung für die Abbildung des Bereiches $\Phi$ auf das Innere des $\zeta$-Einheitskreises zurückgeführt, d. $h$. auf die Untersuchung der Abbildungsfunktion $\zeta\left(\zeta^{\prime}\right)$.

Sei $\lambda$ ein analytisches Begrenzungsstück des Bereichs $\Phi$. Die Funktion $\zeta\left(\zeta^{\prime}\right)$ muß sich dann in jedem Begrenzungspunkte $\zeta_{0}^{\prime}$ von $\lambda$ zunächst stetig verhalten. Denn andernfalls würde man innerhalb $\Phi$ in beliebiger Nähe der Stelle $\zeta_{0}^{\prime}$ Linienstücke beliebig kleiner Länge angeben können, denen in der $\zeta$-Ebene Linien entsprechen würden, die sich gleichmäßig der Peripherie des Einheitskreises annähern und dabei Amplitudenschwankungen hätten, die für alle diese Linien oberhalb einer von Null verschiedenen Schranke $\gamma$ bleiben. Dies bedingt, daß es ein endliches Peripheriestück des $\zeta$-Einheitskreises gibt, längs dessen ganzer Ausdehnung solche Linien in beliebiger Nähe verlaufen würden. Bedenkt man nun, daß die Funktion $\zeta^{\prime}(\zeta)$ innerhalb des $\zeta$-Einheitskreises beschränkt ist, daB ferner die Funktionswerte auf den genannten Linien sich schließlich auf den Wert $\zeta_{0}^{\prime}$ reduzieren, so folgt, daß die Funktion $\zeta^{\prime}(\zeta)$ überhaupt eine Konstante wäre $\left.{ }^{2 a}\right)$. Da dies jedoch nicht der Fall ist, ergibt sich somit die Stetigkeit der Funktion $\zeta\left(\zeta^{\prime}\right)$ und folglich auch der Funktion $\zeta(z)$ längs jedes analytischen Begrenzungsteiles, offenbar auch in den Ecken, die von analytischen Begrenzungsteilen gebildet werden, ferner überhaupt auch für Begrenzungsteile vom Charakter Jordanseher Kurvenstücke.

Wir wollen die angedeutete Verallgemeinerung auf Jordansche Begrenzungsstücke hier nicht weiter verfolgen, verweisen diesbezüglich vielmehr auf unsere oben zitierte Abhandlung. Wir setzen jetzt die Betrachtung für ein aralytisches Begrenzungsstïck in folgender Weise fort.

Die Funktion $\zeta\left(\zeta^{\prime}\right)$, von der wir bereits wissen, daß sie längs eines analytischen Begrenzungsstückes rtetig ist, kann niemals längs eines solchen Teils oder eines Stückes davon konstant sein. Denn dies würde wiederum zur Folge haben, daß die Funktion $\zeta\left(\zeta^{\prime}\right)$ sich überhaupt anf eine Konstante reduziert. Somit wird ein analytisches Begrenzungsstück $\lambda$ des Bereiches $\Phi$ und damit auch ein analytisches Begrenzungsstück $l$ des Bereiches $F$ unter Aufrechterhaltung der Anordnung der Punkte stetig eineindeutig auf ein entsprechendes Stück der Peripherie des $\zeta$-Einheitskreises übertragen.

2a) Siehe meine genannte Abhandlung in Journ. f. Math. 145, S. 213. 
Um nun weiter die Regularität der Abbildungsfunktion $\zeta(z)$ längs $l$ nachzuweisen, gehen wir von dem gewonnenen Ergebnis der Stetigkeit dieser Abbildung aus. Wir machen eine reguläre Hilfsabbiidung eines $l$ einbettenden Flächenteiles, bei der $l$ selbst in ein Stück $B C$ der Achse des Reellen übergeht. Es sei $z^{\prime}(z)$ die Abbildungsfunktion; es sei ferner $A$ ein Punkt innerhalb des Intervalls $B C$. Dann beschreiben wir um $A$ als Mittelpunkt einen kleinen Kreis, der eine ganz in dem gefundenen Flächenstreifen enthaltene Kreisfläche $K$ einschließt. Die obere Halbkreisfläche $K^{\prime}$ ersehemt jetzt auf ein zweieckförmiges Teilgebiet $\varphi$ der $\zeta$-Einheitskreisfläche abgebildet, dessen Begrenzung aus zwei analytischen Linienstücken besteht, einem innerhalb des $\zeta$-Einheitskreises verlaufenden und einem Stück $\beta \gamma$ des $\zeta$-Einheitskreises selbst. Durch lineare Transformation verwandeln wir das Gebiet $\varphi$ in ein anderes $\varphi^{\prime}$, indem wir dem Intervall $\beta \gamma$ ein Intervall $\beta^{\prime} \gamma^{\prime}$ der Achse des Reellen entsprechen lassen. Spiegeln wir $\varphi^{\prime}$ an der Achse des Reellen, so entsteht ein größerer symmetrischer Bereich $\left(\varphi^{\prime}+\varphi^{\prime \prime}\right)$. Es ist dann eine Abbildungsbeziehung zwischen ' der Kreisfäche $K$ und dem Gebiete $\left(\varphi^{\prime}+\varphi^{\prime \prime}\right)$ hergestellt, bei der dem reellen Intervall $B C$ das reelle Intervall $\beta^{\prime} \gamma^{\prime}$ stetig entspricht. Nun kann man aber eine solche Abbildung zwischen $K$ und $\left(\varphi^{\prime}+\varphi^{\prime \prime}\right)$ auch direkt dadurch gewinnen, daß man die Fundamentalabbildung des Bereiches $\left(\varphi^{\prime}+\varphi^{\prime \prime}\right)$ auf die Fläche $K$ vornimmt, wobei auch der Symmetrie genügt werden kann. Diese Abbildung ist innerhalb des reellen Intervalls jedenfalls regulär. Sie muß mit der vorbetrachteten Abbildung übereinstimmend werden, wenn man abgesehen von der bereits richtig beibehaltenen Zuordnung der Intervallendpunkte auch noch von einem inneren Punkte des Intervalls den zugeordneten Punkt in derselben Weise bestimmt wie bei jener Abbildung. Man hat dann nämlich zwei Abbildungen der Fläche $\varphi^{\prime}$ auf die Halbkreisfläche $K^{\prime}$, bei der drei Randpunktpaare in gleicher Weise zugeordnet sind.

\section{Gewöhnliche Randwertaufgabe.}

Es sei nunmehr die gewöhnliche Randwertaufgabe für den Bereich $F$ zu lösen. Wir denken uns dazu die Fläche $F$ zweckmäßig längs $q+2 p$ Querschnitten von Rand zu Rand zu einer einfach zusammenhängenden Fläche $F_{0}$ aufgeschnitten. Die Fläche $F^{(\infty)}$ entsteht durch sukzessive relationenfreie Aneinanderheftung unendlich vieler Exemplare $F_{0}$. Dem Grundexemplar $F_{0}$ entspricht in der $\zeta$-Ebene ein Bereich $\Phi_{0}$ mit $2 p$ Querschnittseiten, die parweise durch hyperbolische lineare Substitutionen einander zugeordnet sind. Diese Substitutionen erzeugen die Fundamentalgruppe $\Gamma$. Bei Ausübung dieser Fundamentalgruppe auf $\Phi_{0}$ ergeben sich unendlich viele Bilder von $\Phi_{0}$, deren auf der Peripherie des $\zeta$-Einheitskreises lie- 
gende Randteile diesen Kreis bis auf unendlicb viele diskret liegende Punkte ausfüllen. Diese Grenzpunkte lassen sich in endlich viele Intervalle von beliebig kleiner Gesamtlänge einschließen, weil die Summe der Längen der Bilder aller Querschnittseiten von $\Phi_{0}$ wegen der eigentlichen Diskontinuität der Gruppe $\Gamma$ auf der Peripherie des Einheitskreises konvergiert ${ }^{3}$ ). Uberpflanzt man jetzt die gegebenen Randwerte des Bereiches $\boldsymbol{F}$ auf dis Peripherie des $\zeta$-Einheitskreises, wo sie in unendlich häufiger Wiederholung gemäß $\Gamma$ erscheinen, sa kann man nunmehr ohne weiteres das Poissonsche Integral für diese Randwerte ansetzen und erhält eine Potentialfunktion innerhalb des $\zeta$-Einheitskreises, die mit Rücksicht auf die Geltung des Unitätssatzes für die Randwertaufgabe (trotz der unendlich vielen Grenzpunkte wegen der Beschränktheit der vorgegebenen Randwerte und wegen der erwähnten Einschließungsmöglichkeit der Grenzpunkte) gegenüber den Substitutionen der Gruppe $\Gamma$ ungeändert bleibt, daher in der Ubertragung auf $F$ eindeutig wird, wie verlangt.

\section{Einführung von polaren, logarithmischen, Areustangens- Unstetigkeiten, von inneren und Rand-Periodizitätsmoduin.}

Schreibt man außer den Randwerten auf $F$ auch noch innere polare oder logarithmische Unstetigkeiten vor, so kann diese Aufgabe nach dem soeben gewonnenen Ergebnis auf den Fall reduziert werden, daß die Randwerte identisch null sind. Nach dieser Reduktion lassen sich dann die betreffenden Potentiale sofort durch unendliche Reihen darstellen. Man sucht im Bereiche $\Phi_{0}$ die entsprechenden Unstetigkeitsstellen auf und schreibt dort die der Ubertragung gemäß bestimmten Unstetigkeiten vor. Es gibt dann eine elementare Potentialfunktion, die-diese Unstetigkeiten und nur diese im Innern des $\zeta$-Einheitskreises bzw: der im folgenden statt der Kreisfiäche setzbaren zugrunde gelegten oberen $\zeta$-Halbebene hat. Diese Potentialfunktion unterwirft man sämtlichen durch die Gruppe $\Gamma$ vorgeschriebenen Verpflanzungen und summiert die so entstehenden unendlich vielen Potentialen. Das Resultat ist ein automorphes Potential, das, auf $F$ überpflanzt, die verlangte Potentialfunktion liefert ${ }^{4}$ ).

Gibt man die Randwerte null und im Innern von $F$ zwei Arcustangens-Unstetigkeiten mit entgegengesetzt gleichen Periodizitätsmoduln vor, wobei man sich zweckmäßig die Fläche $F$ bzw. $F_{0}$ längs einer die Unstetigkeitspunkte verbindenden Linie aufgeschnitten denkt, um die $\dot{P}_{0}$ tentialfunktion eindeutig zu machen, so führt das gleiche Verfahren zum

a) Siehe hierzu Abhandlungen zur Theorie der konformen Abbildung. IV ${ }^{4}, \S 6$, Acta math. 41 (1918), S. 326-328.

4) Siehe hierzu 1. c. Abh. IV der genannten Serie, $\$ \$ 4,6,7$. 
Ziele. Das Elementarpotential in der $\zeta$-Ebene hat die geometrische Bedeutung der Summe der scheinbaren Größe des Bildpunktepaares jener Unstetigkeitsstellen in $\Phi_{0}$ plus der scheinbaren Größe seines Spiegelpaares in bezug auf die Achse des Reellen, gesehen vom Punkte $\zeta$ aus. Das gesuchte Potential wird gefunden als Summe der scheinbaren Größen aller mit den beiden genannten äquivalenten Punktpaare, ebenfalls vom Punkte $\zeta$ aus gesehen.

Um die $2 p+q-1$ auf $F$ existierenden überall endlichen Potentiale erster Art zu gewinnen, hat man einerseits das Potential zu bestimmen, das längs einer Randlinie den konstanten Wert 1, längs der übrigen Randlinien den konstanten Wert 0 hat. Diese Aufgabe subsumiert sich unter die erste oben besprochene Randwertaufgabe, ist aber direkt durch Reihenblddung lösbar. Es ergibt sich eine Darstellung als Summe der scheinbaren Größen von unendlich vielen Fixpunktepaaren (s. L. c. §4). Zweitens muß man ein Potential konstruieren, das längs aller Randlinien den konstanten Wert null annimmt, längs eines vorgegebenen nicht zerfällenden inneren Rückkehrschnittes jedoch einen konstanten Sprung aufweist. Diese Funktion kann man aus Funktionen mit je zwei Arcustangens-Unstetigkeiten gewinnen, indem man längs des Rückkehrschnittes eine endliche Anzahl von Unstetigkeitspunkten einführt und nunmehr je zwei in der geschlossenen Reihe aufeinander folgende dieser Punkte als Paar für zwei entgegengesetzte Arcustangens-Unstetigkeiten benutzt und die erhaltenen Potentiale addiert, wobei die Unstetigkeiten zum Wegfall und der gewünschte Wertesprung längs des geschlossenen Rückkehrschnittes zum Vorschein kommt.

Sind auch Periodizitätsmoduln längs der Randlinien vorgeschrieben, so kann man, um keine Randwerte $z u$ bevorzugen, die Randbedingung „normale Ableitung gleich null“ss) zweckmäßig heranziehen. Sind nun keine inneren Arcustangens-Unstetigkeiten vorgeschrieben, so kann man jetzt die Randperiodizitätsmoduln zum Verschwinden bringen, indem man die zu den oben konstruierten Potentialen erster Art mit konstanten Randwerten konjugierten Potentiale, die durch die betreffenden unendlichen Reihen mitgeliefert werden, herstellt und eine geeignete Kombination derselben von der zu bestimmenden Funktion in Abzug bringt. Sind jedoch auch Arcustangens-Unstetigkeiten in endlicher Zahl im Innern gegeben; so kann im besonderen die Summe der dazugehörenden Periodizitätsmoduln null sein. Dann ist auch die Summe der Randperiodizitätsmoduln gleich null und man kann alle Randperiodizitätsmoduln zum Wegfall bringen, wenn man erstens eine lineare Kombination oben genannter

3) Vgl. \$ $81 . \mathrm{c.}$ 
konjugierter Potentiale von der zu bestimmenden Funktion abzieht und weiter eine lineare Kombination von Potentialen mit je zwei ArcustangensUnstetigkeiten. Ist aber die Summe der zu den Arcustangens-Unstetigkeiten gehörenden Periodizitätsmoduln nicht gleich null,'so wird man durch das Abzugsverfahren auf die Bestimmung eines Potentiales geführt, das nur eine Arcustangens-Unstetigkeit im Innern und einen gleich großen Randperiodizitätsmodul besitzt. Eine solche Funktion mit der normalen Ableitung Null am Rande läßt sich dann aber als Summe von scheinbaren Größen in der $\zeta$-Ebene aufbauen. Die Stammgröße ist hierbei die scheinbare Größe desjenigen Punktepaares, das vom korrespondierenden Punkte der Unstetigkeitsstelle und seinem Spiegelpunktbilde in bezug auf die Achse des Reellen der $\zeta$-Ebene gebildet wird.

\section{Bildung einer zu $F$ gehörenden reellen algebraischen Kurve.}

Die vorstehenden Entwicklungen geben auch die Grundlage ab, um dem Bereiche $F$ eine reelle algebraische Kurve $(x, y)$ in demselben Sinne zuzuordnen, wie dies Schottky in seiner Dissertation (Journ. f. Math. 83) für endlich-vielfach zusammenhängende schlichte Bereiche gatan hat. Die Größen $x$ und $y$ werden als zwei solche Funktionen der Variablen $z$ bestimmt, die in $F$ abgesehen von endlich vielen Polen eindeutig und regulär sind, ferner am Rande nur reelle Werte annehmen, ferner die Eigenschaft haben, daß alle übrigen Funktionen $w(z)$ mit denselben genannten Eigenschaften sich rational durch $x$ und $y$ ausdrücken lassen. Um die Funktionen $x$ und $y$ zu bilden, genügt es offenbar, Potentiale mit nur polaren Unstetigkeiten heranzuziehen, aus denen sich durch lineare Kombination sofort solche Potentiale ergeben, deren konjugierte Potentiale sicher eindeutig sind. Man kann zwecks Bildung solcher Funktionen auch mit Poincaréschen $\theta$-Quotienten operieren. Hat man etwa auf solche Weise eine Kurve $(x, y)$ gewonnen und damit den zur Fläche $F$ gehörenden reellen algebraischen Funktionenkörper, so kann man natürlich auch von hier aus die Bildung der Potentiale bzw. deren Differentiale nach bekannten Methoden vornehmen.

\section{Behandlung gesehlossener Riemannscher Flächen.}

Es sei $F$ eine geschlossene Riemannsche Fläche. Hier bieten sich der'Behandlung zwei Wege. Entweder man konstruiert zunächst zu $F$ gehörige algebraische Funktionen und damit eine zugehörige algebraische Kurve $(x, y)$, indem man die Fläche $F$ einer Fundamentalabbildung (zweckmäßig mit Relativverzweigung) ${ }^{6}$ ) unterwirft und dann Poincaré-

i) Siehe „Abhandlungen zur Thenrie der konformen Abbildung. $1 I^{“}$, $\$ 8$, Acta math. 40 (1916), S. $287-290$. 
254 P. Koebe. Fundamentalabbildung a. Potentialbestimmung Riemannscher Flächen:

sche $\Theta$-Quotienten in Ansatz bringt, darnach die gewünschten Potentiale nach bekannten Methoden herstellt; oder aber, man verwandelt die Fläche $F$ zunächst in eine berandete Fläche $F^{\prime}$ durch Entfernung etwa einer kleinen Kreisscheibe $k$. Mit den zu $F^{\prime}$ gehörenden Potentialen kann man dann unschwer durch Grenzïbergang, indem man $k$ sich auf einen Punkt reduzieren läßt, zu den Potentialen der Fläche $F$ gelangen, wie dies von mir in $\$ 4$ der Abh. III der wiederholt genannten Serie in einem bestimmten Falle dargelegt ist ${ }^{7}$ ). Es macht dabei nichts aus, auch mehrere Offnungen gleichzeitig einzuführen. Von diesen letzten Bemerkungen kann man zweckmäßig Gebrauch machen, indem man aus der Riemannschen Fläche $F$ durch alle Blätter hindurch Kreisscheiben ausgestanzt denkt, so, daß dabei sämtliche Windungspunkte zum Fortfall kommen. Die zur so entstandenen offenen Fläche $F$ gehörige Fundamentalveränderliche ohne relative Verzweigung ist dann identisch mit der Fundamentalveränderlichen des schlichten mehrfach zusammenhängenden Bereiches, ïber dem die Fläche $F$ ausgebreitet ist $\left.{ }^{8}\right)$.

Schließlich sei noch bemerkt, daß jede geschlossene Riemannsche Fläche $F$ durch elementare eineindeutige Abbildung in eine andere geschlossene Riemannsche Fläche mit lauter reellen Windungspunkten verwandelt werden kann. Ist nämlich etwa $a+b i$ ein nichtreeller Windungspunkt, so wird die Fläche $F$ durch die Transformation $(z-a)^{2}=z^{\prime}$ auf eine andere Fläche abgebildet, deren Anzahl nichtreeller Windungspunkte um mindestens eine Einheit verringert worden ist. Durch wiederholte Anwendung solcher Transformationen kommt man also schließlich zu einer Fläche mit nur reellen Windungspunkten. Die Gesamtzahl dieser Windungspunkte hat sich dabei im allgemeinen vergrößert ${ }^{9}$ ).

7) Journ. f. Math. 147 (1917), S. 77-83.

8) Diese Fundamentalveränderlichen werden in $\mathrm{Abh}$. II der genannten Serie mittels eines Schmiegungsverfahrens bestimmt.

9) Vgl, auch Poincaré in Acta math. \$ (1884), S. 246-250. 